\title{
The effect of vitamin D3 and thyroid hormones on the capillaroscopy-confirmed microangiopathy in pediatric patients with a suspicion of systemic connective tissue disease-a single-center experience with Raynaud phenomenon
}

\author{
Katarzyna Kapten $^{1}$ D $\cdot$ Krzysztof Orczyk $^{2}$ (D) Elzbieta Smolewska ${ }^{2}$ D \\ Received: 24 March 2021 / Accepted: 9 June 2021 / Published online: 16 June 2021 \\ (c) The Author(s), under exclusive licence to Springer-Verlag GmbH Germany, part of Springer Nature 2021
}

\begin{abstract}
Raynaud phenomenon (RP) may be the first manifestation of a systemic connective tissue disease (SCTD). Early detection of dysfunction of small vessels called microangiopathy is essential for the diagnostic process. The focus of this single-center, retrospective study was to investigate the potential dependencies between microvascular image and laboratory markers measured in children with RP. The study analyzed the nail-fold video-capillaroscopy (NVC) findings and laboratory results of 81 children between the ages 6 and 17 who were referred to pediatric rheumatologist with a suspicion of SCTD. Out of 52 patients presenting with RP at the time of evaluation, abnormalities in capillary microscopic imaging were found in 34 . NVC findings were then compared to levels of specific biomarkers in serum. Vitamin D3 serum levels have been significantly decreased in patients with $\mathrm{RP}(23.4 \mathrm{ng} / \mathrm{mL} \pm 8.76$ vs. $30.0 \mathrm{ng} / \mathrm{mL} \pm 12.66, P=0.0148)$. There were positive significant correlations between levels of vitamin D3 and acute-phase reactants in serum, such as C-reactive protein $(P=0.0292)$. Furthermore, free thyroxine levels (fT4) in patients with both RP $(P=0.0126)$ and micro-angiopathy $(P=0.05496)$ persisted in the lower range of the normal limit $(<1.0 \mathrm{ng} / \mathrm{dL})$. Regular oral supplementation of vitamin D3 should be always considered in children with RP if deficiency is found. Additionally, low fT4 level $(<1.0 \mathrm{ng} / \mathrm{dL})$ should be considered as an indication to perform NVC in patients suspected of SCTD even when they do not present RP.
\end{abstract}

Keywords Capillaroscopy $\cdot \mathrm{fT} 4 \cdot$ Microangiopathy $\cdot$ Raynaud phenomenon $\cdot$ Thyroid hormones $\cdot$ Vitamin D3

$\begin{array}{ll}\text { Abbreviations } \\ \text { ANA } & \text { Antinuclear antibodies } \\ \text { ANCA } & \text { Antineutrophil cytoplasmic antibodies } \\ \text { CALs } & \text { Coronary arterial lesions } \\ \text { CRP } & \text { C-reactive protein } \\ \text { ESR } & \text { Erythrocyte sedimentation rate }\end{array}$

Elzbieta Smolewska

e.smolewska@wp.pl

Katarzyna Kapten

katarzynakapten@gmail.com

Krzysztof Orczyk

krzysztof.orczyk@umed.lodz.pl

1 Department of Pediatric Cardiology and Rheumatology, Central Teaching Hospital of Medical University of Lodz, Lodz, Poland

2 Department of Pediatric Cardiology and Rheumatology, Medical University of Lodz, Sporna 36/50, 91-738 Lodz, Poland
fT4 Free thyroxine

IL Interleukin

KD Kawasaki disease

MCTD Mixed connective tissue disease

MIS-C Multisystem inflammatory syndrome in children

NVC Nailfold videocapillaroscopy

PLT Platelet count

RP Raynaud phenomenon

SCTD Systemic connective tissue disease

SLE Systemic lupus erythematosus

SSc Systemic Sclerosis

TNF- $\alpha$ Tumor necrosis factor alpha

TSH Thyroid stimulating hormone 


\section{Background}

Raynaud phenomenon (RP) is a condition, which first description dates back to 1860 s, with a typical onset between the ages of 15 and 30 years. Nevertheless, it can be manifested in children as early as in the first decade of life, with higher prevalence in females than in males [1]. Having a very distinctive triphasic clinical pattern, including sudden pallor of the fingers, followed by a cyanotic phase and ending with redness due to reactive hyperemia, it remains one of the most common symptoms in rheumatic diseases. The pathophysiology of RP, however, is a highly complex mechanism of neurogenic, inflammatory and immune responses [2]. Very promising research, such as its association with the release of Endothelin-1 and nitric oxide [3, 4], facilitates a better understanding of the pathophysiology of RP (Fig. 1) and therefore can improve its treatment. It is crucial for clinical practice to distinguish patients with primary RP, an isolated symptom with good prognosis and little morbidity, from patients with secondary RP, which is merely a manifestation of an underlying disease [5]. Interestingly, Piotto et al. [6] reported that out of 40 children who were initially diagnosed with primary RP, three eventually developed an autoimmune disease: (1) mixed connective tissue disease (MCTD); (2) hypothyroidism; (3) systemic lupus erythematosus (SLE). In all aforementioned cases, systemic connective tissue disease (SCTD) was excluded on initial visit after a thorough investigation. One of these patients presented scleroderma-pattern micro-angiopathy at study baseline, the other one presented abnormal nail-fold videocapillaroscopy (NVC) pattern on the re-evaluation, and the remaining one did not present it at all. Thus, the authors underlined the necessity of further studies to assess NVC as a tool for identifying patients with higher risk of developing secondary RP.

Vitamin D3 deficiency is an important comorbidity in children diagnosed with rheumatic diseases. While its main physiological roles are the homeostasis perseverance of calcium levels in serum and bone metabolism, vitamin D3 deficiency is associated with higher prevalence of several entities including asthma [7] or acute respiratory tract infections [8] and may play a pivotal role in their management. Studies conducted on the role of vitamin D3 as a biomarker for cardiovascular diseases in children, such as Kawasaki

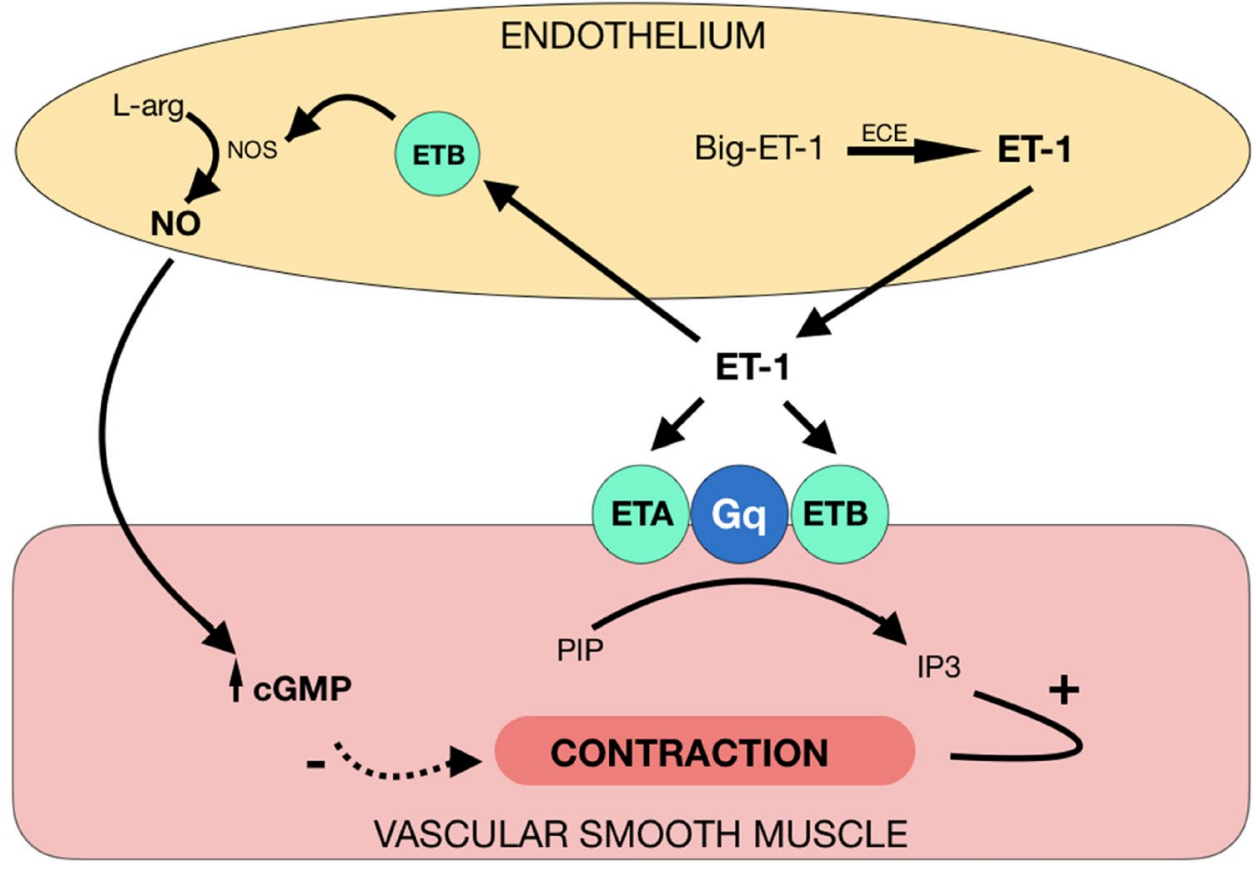

Fig. 1 Pathomechanism of vasoconstriction in patients with Raynaud phenomenon. The figure illustrates Endothelin-1 (ET-1) receptors in vascular smooth muscle and their interactions with nitric acid (NO). ET-1 is produced from big-ET-1 catabolized by the ET-converting enzyme (ECE) expressed in endothelial cells. ET-1 binds to ETA and ETB receptors, both of which share the same G-protein (Gq) signaling pathway. Activation of either receptor leads to processing the molecule phosphatidylinositol 4,5-bisphosphate (PIP) into inositol trisphosphate (IP3) on vascular smooth muscle facilitating the muscle contraction. ETB receptors are expressed on both endothelial cells and smooth muscle, mediating vasoconstriction. Binding ET-1 to endothelial ETB receptors stimulates the activation of nitric oxide synthase (NOS) that converts L-arginine ( $\mathrm{L}$-arg) to nitric oxide (NO), which diffuses into the vascular smooth muscle cells to activate formation of cyclic guanosine monophosphate (cGMP), which signals smooth muscle relaxation and leads to vasodilation 
disease (KD), orthostatic intolerance and hypertension [9] evoke further questions on its role and further magnitude in inflammatory and autoimmune reactions. However, there are some inconsistent data on the possible anti-inflammatory role of vitamin D3. While Stagi et al. [10] had shown that patients with KD had severely reduced serum levels of 25(OH)D3 compared with control group, Chen et al. [11] reported contradictory findings. In the latter study, patients treated for KD had higher vitamin D3 serum levels than the healthy controls. It was already proven that vitamin D3 deficiency is correlated with increased levels of proinflammatory cytokines, such as tumor necrosis factor alpha (TNF- $\alpha$ ), interleukin (IL)-6 and IL-1 $\beta$, as well as having a crucial role in the maturation of human monocytes [12]. Moreover, numerous studies confirmed an inverse correlation between vitamin D3 and both C-reactive protein (CRP) level [13-15] and platelet count (PLT) [16] in healthy individuals. Regarding the importance of vitamin D3 in the treatment of rheumatic diseases, Hélou et al. [17] reported a significant alleviation of symptoms of RP in adults after high-dose vitamin D3 supplementation, the results were collected in a double blinded, placebo-controlled study. Moreover, Szymańska-Kałuża et al. [18] observed decreased levels of active metabolite of vitamin D3 in children diagnosed with juvenile idiopathic arthritis, stressing the significance of routine evaluation of vitamin D3 levels in these patients. Furthermore, Saggese et al. in the "state of the art" review [19] on vitamin D3 in childhood and adolescence postulated the need for more randomized controlled trials regarding vitamin D3 as a potentially crucial factor in the global health of children and adolescents.

While there is a clear and widely acknowledged link between the majority of present-day thyroid diseases and autoimmune mechanisms, the association between thyroid hormones and specific rheumatic diseases is still mostly based on singular case studies. There has been, however, a positive effect of thyroid hormone substitution therapy in patients with RP [20, 21]. Shagan et al. [22] reported the rapid improvement of RP after hormone therapy in patients with non-autoimmune hypothyroidism. Conversely, Withrington et al. [23] observed the return of normal thyroid function after steroid therapy without hormone treatment in a patient diagnosed with Hashimoto disease and MCTD with RP. According to Ciaffi et al. [24], abnormalities in capillaroscopic image can be related to the number of comorbidities (other than SCTDs) that can possibly affect the microcirculation and may require performing NVC. A further research on the impact of thyroid hormones in regard to microcirculation may bring useful information regarding their possible application to the diagnosis and treatment of rheumatic diseases.

NVC is the most commonly used imaging technique for the diagnosis of RP and various connective tissue disorders.
As stated in the survey conducted by Ingegnoli et al. [25], NVC was by far the most familiar technique among rheumatologists and it was most widely used in their everyday practice. According to Tavacol et al. [26], this non-invasive and safe technique is a fairly sensitive and highly specific tool for differentiating between primary and secondary RP and furthermore diagnosing and monitoring a number of connective tissue disorders. Furthermore, there is no significant difference in diagnostic value of NVC between adults and children. Additionally, Schonenberg-Meinema et al. [27] postulated that specific nail-fold capillary abnormalities detected in NVC should be considered as a new organ involvement marker in the assessment of disease activity in children diagnosed with SLE. As suggested by TorrenteSegarra et al. [28], the broad use of NVC in children suspected of SCTD can help rheumatologist properly address patients with positive titer of antinuclear antibodies (ANA), irrespectively of the presence of RP.

The aim of the study was to answer the following questions: (1) Is it reasonable to screen patients with RP for specific comorbidities including hypothyroidism and vitamin D3 deficiency? (2) Should we perform NVC in patients with aforementioned comorbidities when they do not present RP? To address these questions, the authors assessed the dependencies between blood test results, involving serum levels of vitamin D3 and thyroid hormones, and the capillaroscopic image of the microcirculation in patients with suspected SCTD, including children with RP.

\section{Methods}

The study involved 81 patients with a suspicion of SCTD who were admitted to the Department of Pediatric Cardiology and Rheumatology, Medical University of Lodz, Poland, between January 2019 and September 2020. Active $\mathrm{RP}$ was present in 52 out of 81 patients participating in the research. Within our study group 11 children suffered from SLE, one patients with Sjögren syndrome, one with KD, one with vasculitis and one with MCTD. All the patients that were included in the study underwent NVC. In case in which the examination was performed more than once (which applied to 10 patients), the earliest one was taken under consideration. Patients records were subsequently analyzed to build a comprehensive database containing: age, sex, presence of active RP; micro-angiopathic findings in capillaroscopic imaging (in particular mega-capillaries, micro-hemorrhages and branched loops); autoimmune biomarkers in serum, including ANA, anti-neutrophil cytoplasmic antibodies (ANCA) and antiphospholipid antibodies, complement components; inflammatory markers, including CRP, erythrocyte sedimentation rate (ESR) and PLT; thyroid hormones, specifically thyroid stimulating hormone (TSH) 
and free thyroxine (fT4); coagulation markers (activated partial thromboplastin time with international normalized ratio); vitamin D3 levels. As proposed by Okazaki et al. [29], we used the cut-off values for serum $25(\mathrm{OH}) \mathrm{D}$ insufficiency between 20 and $30 \mathrm{ng} / \mathrm{mL}$ and we defined vitamin D3 deficiency as levels less than $20 \mathrm{ng} / \mathrm{mL}$. We defined the low range of normal limit for fT 4 at $<1 \mu \mathrm{IU} / \mathrm{mL}$, with laboratory norm $0.510-4.82 \mu \mathrm{IU} / \mathrm{mL}$. The collected data were then compared between two groups: (1) children presenting active RP (called RP+) and (2) other patients suspected of SCTD with no symptoms of RP (called RP-).

All statistical calculations were carried out using Statistica 13.1 software (Statsoft Polska, Krakow, Poland). The values were expressed as mean \pm standard deviation (SD). The normality of the continuous variables was tested using Shapiro-Wilk test. The Mann-Whitney $U$ test and Kruskal-Wallis test were used for group comparisons. Relations between categorical variables were assessed using Pearson's chi-squared test. $P$ values lower than 0.05 were considered significant.

\section{Results}

\section{General characteristics of the study group}

General characteristics of the study group are presented in Table 1. Abnormalities in NVC image were detected in 34 children, while micro-angiopathy was diagnosed in 18 of them, and 16 needed further observation towards micro-angiopathy.

\section{Vitamin D3}

Vitamin D3 serum levels have been significantly decreased in $\mathrm{RP}+$ patients $(23.4 \mathrm{ng} / \mathrm{mL} \pm 8.76$ vs. $30.0 \mathrm{ng} / \mathrm{mL} \pm 12.66$, $P=0.0148$ ) when compared to RP- group (Fig. 2). Insufficient serum $25(\mathrm{OH}) \mathrm{D}$ levels $(20-30 \mathrm{ng} / \mathrm{mL})$ were found in $36.5 \%$ of RP+ patients, while vitamin D3 deficiency was detected in $28.8 \%$ of RP+ patients. Vitamin D3 deficiency or insufficiency was diagnosed only in $27.5 \%$ and $17.2 \%$ of RP children, accordingly. There was no significant difference in frequency of RP between patients with insufficient and deficient levels of vitamin D3 in serum.

\section{Acute-phase reactants}

No correspondence between the levels of acute-phase proteins in serum and RP or abnormal capillaroscopic findings was found. Nonetheless, there was a positive correlation between levels of vitamin D3 in serum and acute-phase
Table 1 General characteristics of the study group

\begin{tabular}{lll}
\hline & $\mathrm{RP}+(n=52)$ & $\mathrm{RP}-(n=29)$ \\
\hline Male/female & $12 / 40$ & $6 / 23$ \\
Age on examination (years) & $15.29 \pm 1.64$ & $12.10 \pm 4.12$ \\
NVC image & & \\
$\quad$ Microangiopathy & $15(28.8 \%)$ & $3(10.3 \%)$ \\
Observation towards microangiopa- & $12(23.1 \%)$ & $4(13.8 \%)$ \\
$\quad$ thy & $6(11.5 \%)$ & $0(0 \%)$ \\
Mega-capillaries & $17(32.7 \%)$ & $8(27.6 \%)$ \\
Micro-hemorrhages & $19(36.5 \%)$ & $10(34.5 \%)$ \\
Branched loops & & \\
Laboratory features & $19(36.5 \%)$ & $8(27.5 \%)$ \\
25(OH)D 20-30 ng/mL & $15(28.8 \%)$ & $5(17.2 \%)$ \\
25(OH)D $<20$ ng/mL & $23.39 \pm 8.76$ & $24.88 \pm 12.66$ \\
Mean 25(OH)D value & $27(51.9 \%)$ & $6(20.7 \%)$ \\
fT4<1.0 ng/dL & $0.944 \pm 0.106$ & $0.977 \pm 0.179$ \\
Mean fT4 value & $3(5.8 \%)$ & $3(10.3 \%)$ \\
Presence of ANCA & $18(34.6 \%)$ & $20(69.0 \%)$ \\
Presence of ANA & &
\end{tabular}

Values presented as mean \pm standard deviation (SD)

$A N A$ antinuclear antibodies, ANCA anti-neutrophil cytoplasmic antibodies, $N V C$ nail-fold video-capillaroscopy, fT4 free thyroxine, $R P$ Raynaud phenomenon

reactants. Relations to ESR, CRP measured values and PLT were all statistically significant $(P=0.0292, P=0.0332$ and $P<0.001$, respectively). Patients with normal vitamin D3 levels had significantly higher values of PLT count (Fig. 3).

\section{Thyroid hormones}

Patients presenting RP were found to have levels of fT4 in serum in the lower range of the normal limit $(<1.0 \mathrm{ng} /$ $\mathrm{dL}, P=0.0126$ ). Additionally, decreased levels of fT4 were associated with the presence of capillaroscopy-confirmed microangiopathy $(P=0.05496)$. While no concurrent relation between RP and TSH levels was discovered, higher TSH levels were associated with the presence of branched loops in capillaroscopy imaging. However, it reached only marginal significance $(P=0.0526)$.

\section{Additional findings}

ANCA did not appear to be an efficient marker of microangiopathy in the study group, since they were found in only six children. Furthermore, no correspondence between microvascular findings and antiphospholipid antibodies, ANA or serum levels of complement components have been found.

While the majority of patients in the study group were female, males qualified for NVC were more likely to have 
Fig. 2 A comparison between levels of vitamin D3 among Raynaud phenomenon positive and negative patients

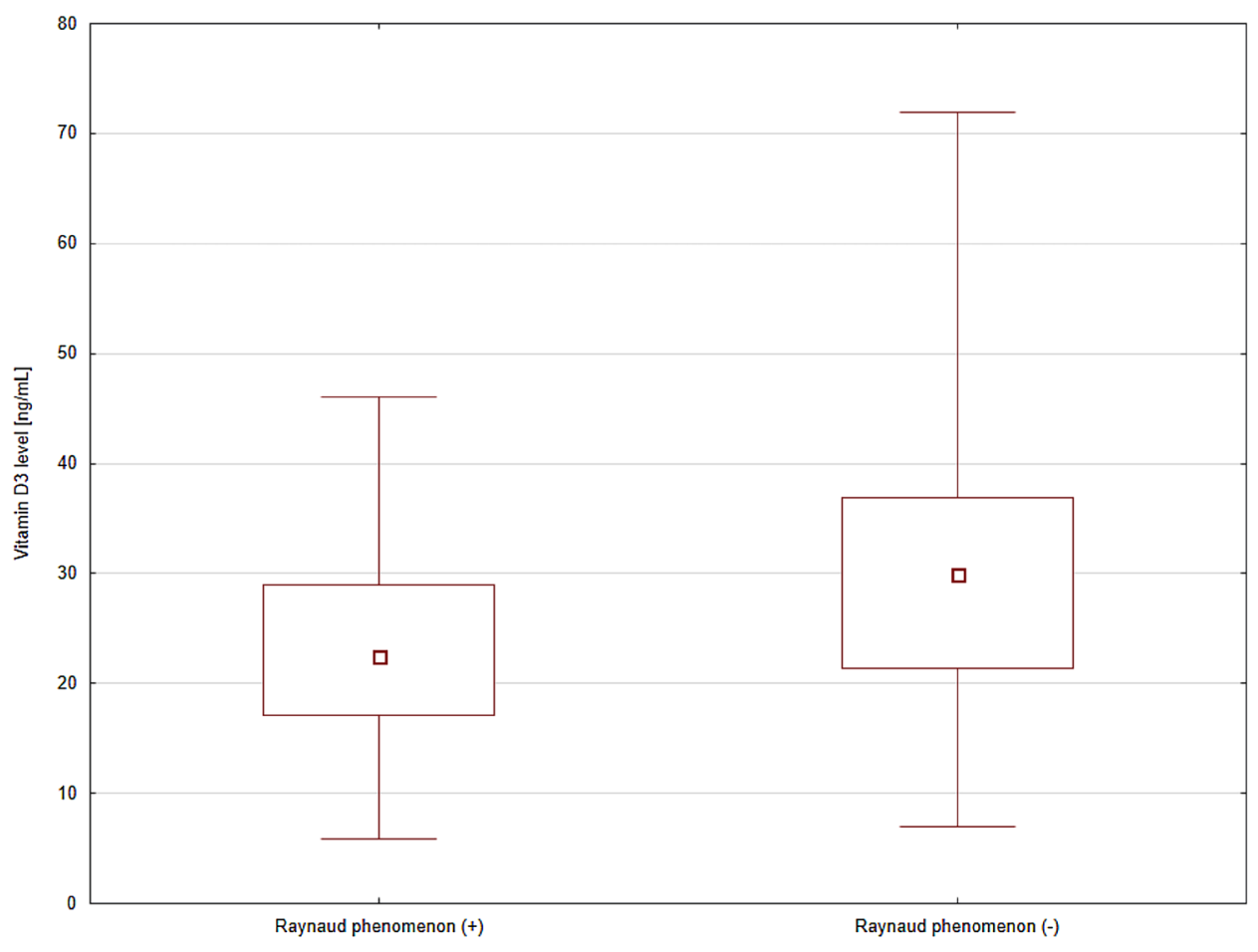

Fig. 3 A comparison between platelet count in patients with levels of vitamin D3 $<30 \mathrm{ng} / \mathrm{mL}$ and patients with vitamin $\mathrm{D} 3$ levels $>30 \mathrm{ng} / \mathrm{mL}$. PLT platelet count

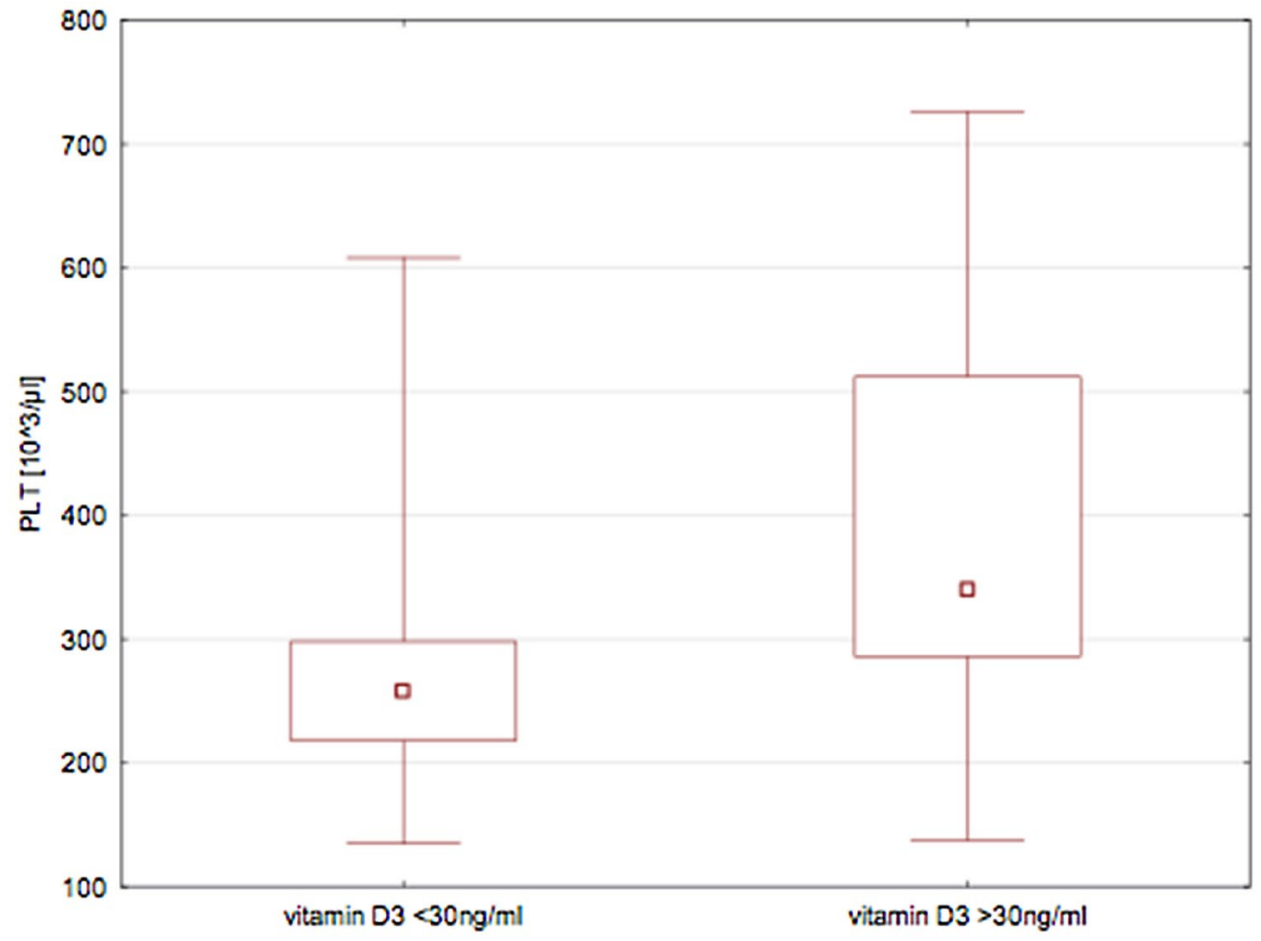

abnormal microcirculation image, with 10 out of 18 (55.6\%) male patients having abnormal capillaroscopic findings, and only 24 out of 63 (38.1\%) females being suspected of microangiopathy following NVC. However, none of the results associated with the gender of the patients reached statistical significance. 


\section{Discussion}

While the recent study analyzed various biological markers, the levels of both vitamin D3 and thyroid hormones were the primary focus of our study, since their clinical value appeared to be the most relevant. The major findings of the study include: observation of the differences of vitamin D3 levels between analyzed groups of patients; surprising irrelevance of inflammatory markers levels in patients with RP; potential importance of fT4 levels in children with RP. The strengths of the study must also include the original approach to the well-established algorithms regarding RP in scleroderma in adults, by performing a study solely on the cohort of pediatric patients.

$\mathrm{NVC}$ has been proven to be an invaluable tool in timely detection and monitoring of rheumatic diseases [26, 30]. The relations between the microcirculation image and the levels of numerous biomarkers in blood serum, alongside the clinical presentation of the disease can maximize the effectiveness in the evaluation of patients, both suspected and already diagnosed with SCTD. Moreover, NVC can be successfully applied to a much wider spectrum of diseases, including one of the most recent and severe entities called SARS-CoV-2-associated multi-system inflammatory syndrome in children (MIS-C) [31]. NVC abnormalities detected in MIS-C were proving microcirculatory dysfunction possibly related to the acute inflammatory reaction within the course of the disease.

Serum levels of vitamin D3 were significantly lower in patients with active RP when compared to children suspected of SCTD but not presenting symptoms of RP. These results are consistent with the study of Hélou et al. [17] who evaluated the improvement in self-judgment of RP symptoms in adults after vitamin D3 supplementation. Nevertheless, no comparable prospective study involving children and adolescents have been performed. Unlike Sampaio-Barros et al. [32], who found negative association between vitamin D3 levels and NVC abnormalities (such as presence of avascular areas and diffuse devascularization), we did not prove significant differences in vitamin D3 levels between patients with NVC-confirmed microangiopathy and children with normal microcirculation. The recent study is concordant with findings of Trombetta et al. [33] and Groseanu et al. [34], who postulated an association with organ involvement in patients with Systemic Sclerosis (SSc) and consequently, a more severe course of disease in patients with decreased vitamin D3 values. However, they also did not find statistical significance between vitamin D3 levels and NVC patterns. While there is no proven correlation between vitamin D3 levels and microcirculation pathologies in rheumatic diseases, numerous research regarding the significance of vitamin D3 levels involving mainly patients with vascular complications of diabetes mellitus [35-37] and cardiovascular diseases $[8,38]$ prove that further studies in this field seem to be a necessity.

The association of lower levels of fT4 hormone with both RP and capillaroscopy-confirmed microangiopathy supports the previous findings of Shagan et al. [20, 22] who suggested that RP may be an early clue to the diagnosis of hypothyroidism. Moreover, the hormone replacement therapy proved to be, on its own, a highly successful treatment in alleviating the symptoms of RP in hypothyroid patients. These results are also consistent with the findings of Molnár et al. [39] who claimed that low fT3 levels may affect the microcirculation in SSc. Furthermore, Gordon et al. [40] revealed a wide range of thyroid abnormalities found in patients with fatal cases of SSc. Hence, it is crucial to incorporate regular and more thorough monitoring of the thyroid function in patients that are being diagnosed towards SCTD. Moreover, with very promising results of thyroid hormone replacement therapy in alleviating the symptoms of RP [21], further research and subsequent revision of cut-off values of free thyroid hormones levels, may indicate the need for treatment in specific cases of severe RP.

Levels of acute-phase reactants in serum, as well as the presence of autoantibodies, did not correspond with the abnormalities in capillaroscopic image, nor the clinical presentation of RP. Such results were also postulated by Martinis et al. [41] in the study assessing patients with anorexia nervosa presenting symptoms of RP and exhibiting NVC findings typical for SCTD. Similarly to the recent study, no significant association were found between capillaroscopic patterns and ESR, CRP or autoantibodies. However, there was a significant positive correlation between the levels of vitamin D3 and acute-phase proteins. The recent findings are not consistent with the majority of research studying those dependencies [13-16], stating an inverse relation between levels of vitamin D3 and CRP or PLT count. These conflicting results may be due to a highly diversified group of patients in the recent study, who were solely suspected of SLE and eventually only some of them were diagnosed with SCTD. A more homogenous group of patients, that beforehand underwent a thorough examination, excluding other plausible causes for presenting the symptoms of collagenosis, may provide more comprehensive data. However, the current study may support the findings of Chen et al. [11] postulating a positive correlation between higher levels of vitamin D3 and development of coronary arterial lesions (CALs) in pediatric patients with KD. This association was attempted to be explained by an intense inflammatory response in patients with CALs, which may subsequently promote elevated vitamin D3 release. However, neither CRP nor ESR levels were measured in that study. The lack of 
corresponding research involving patients with rheumatic diseases necessitates further investigations in this topic.

Summing it up, NVC is an invaluable tool in timely detection and monitoring of rheumatic diseases. It facilitates the diagnostic process in a multitude of conditions, even beyond the scope of SCTDs. Vitamin D3 deficiency is associated with the clinical presentation of active RP; however, its full effect on microcirculation is yet to be discovered. Low levels of thyroid hormones proved to be associated with both RP and capillaroscopy-confirmed microangiopathy. A significant positive correlation between the levels of vitamin D3 and acute-phase reactants found in the study requires further investigation before drawing long-term conclusions.

The major limitation of the present research was the relative inconsistency of the study group and a small sample size, which allowed to find the trends rather than draw highly detailed conclusions. Expanding the study group and collecting comprehensive data from a larger number of patients would yield the most reliable results. Additionally, a randomized double-blind control study with high-dose vitamin D3 supplementation may lead to the establishment of solid recommendations in the treatment of RP. Regarding the diagnostic methods used in the study, all NVCs were performed by the same physician to maintain the reproducibility of the examination technique. However, the collected database did not involve the extrinsic factors including variable weather conditions, patients' preparation for NVC (like resting in room temperature for an adequate period of time) or exposure to cigarette smoke.

\section{Conclusion}

Children diagnosed towards SCTD should have a routine evaluation of their vitamin D3 levels in serum, and a proper supplementation should be considered when a deficiency is observed. Low levels of fT4 $(<1.0 \mathrm{ng} / \mathrm{dL})$ in pediatric patients suspected of SCTD should be an indicator for performing NVC together with regular monitoring of thyroid hormones levels. Assessing NVC patterns in children with lower levels of fT4, irrespectively of the presence of RP, may benefit the patients, that otherwise might have not been qualified for NVC. There is a need for more comprehensive research studying the role inflammatory markers and their correlation to vitamin D3 levels in patients with rheumatic diseases

Author contributions KK collected the data, collected literature, executed the study and drafted the manuscript. KO proposed the study design, performed NVC in all patients, executed statistical analysis, provided images and participated in drafting the manuscript. ES supervised the study and contributed to the overall study design and to the manuscript preparation. All authors revised and approved the final version of the manuscript and agreed to be accountable for all aspects of the work, ensuring its accuracy and integrity.

Funding The study was supported by the Medical University of Lodz, Poland (Grant No. 503/8-000-01/503-81-001-19-00).

Data availability The data used to support the findings of this study are included within the article. The supplementary data are available from the corresponding author upon request.

\section{Declarations}

Conflict of interest The Dino-Lite Capillaryscope 200 Pro was donated by AbbVie Pharmaceutical Research \& Development. The authors declare no other competing interests.

Ethical approval The study does not fulfill the criteria of medical experiment therefore, according to Good Clinical Practice and Polish law, it was not applicable for the Bioethics Committee.

Consent to participate Not applicable.

Consent for publication Not applicable.

\section{References}

1. Temprano KK (2016) A review of Raynaud's disease. Mo Med 113:123-126

2. Walecka I, Malewska A, Roszkiewicz M, Wieczorek M, Lagun Z, Szymanska Z (2017) Raynaud's phenomenon-the clinical picture, treatment and diagnostics. Acta Angiologica 23:29-33. https://doi.org/10.5603/AA.2017.0003

3. Sulli A, Soldano S, Pizzorni C, Montagna P, Secchi ME, Villaggio B, Seriolo B, Brizzolara R, Cutolo M (2009) Raynaud's phenomenon and plasma endothelin: correlations with capillaroscopic patterns in systemic sclerosis. J Rheumatol 36:1235-1239. https://doi.org/10.3899/jrheum.081030

4. Latuskiewicz-Potemska J, Chmura-Skirlinska A, Gurbiel RJ, Smolewska E (2016) Nailfold capillaroscopy assessment of microcirculation abnormalities and endothelial dysfunction in children with primary or secondary Raynaud syndrome. Clin Rheumatol 35:1993-2001. https://doi.org/10.1007/s10067-016-3340-8

5. Musa R, Qurie A (2020) Raynaud disease. StatPearls (Internet). StatPearls Publishing, Treasure Island

6. Piotto DG, Hilário MO, Carvalho NS, Len CA, Andrade LE, Terreri MT (2013) Prospective nailfold capillaroscopy evaluation of Raynaud's phenomenon in children and adolescents. Acta Reumatol Port 38:114-121

7. Litonjua AA (2019) Vitamin D and childhood asthma: causation and contribution to disease activity. Curr Opin Allergy Clin Immunol 19:126-131. https://doi.org/10.1097/ACI.0000000000 000509

8. Martineau AR, Jolliffe DA, Greenberg L, Aloia JF, Bergman P, Dubnov-Raz G, Esposito S, Ganmaa D, Ginde AA, Goodall EC, Grant CC, Janssens W, Jensen ME, Kerley CP, Laaksi I, Manaseki-Holland S, Mauger D, Murdoch DR, Neale R, Rees JR, Simpson S, Stelmach I, Kumar GT, Urashima M, Camargo CA, Griffiths CJ, Hooper RL (2019) Vitamin D supplementation to prevent acute respiratory infections: individual participant data meta-analysis. Health Technol Assess 23:1-44. https://doi.org/10. $3310 /$ hta23020 
9. Xu WR, Jin HF, Du JB (2017) Vitamin D and cardiovascular risk in children. Chin Med J (Engl) 5(130):2857-2862. https://doi.org/ 10.4103/0366-6999.215500

10. Stagi S, Rigante D, Lepri G, Cerinic MM, Falcini F (2016) Severe vitamin D deficiency in patients with Kawasaki disease: a potential role in the risk to develop heart vascular abnormalities? Clin Rheumatol 35:1865-1872. https://doi.org/10.1007/ s10067-015-2970-6

11. Chen YL, Wang JL, Li WQ (2014) Prediction of the risk of coronary arterial lesions in Kawasaki disease by serum 25-hydroxyvitamin D3. Eur J Pediatr 173:1467-1471. https://doi.org/10.1007/ s00431-014-2346-y

12. Di Rosa M, Malaguarnera G, De Gregorio C, Palumbo M, Nunnari G, Malaguarnera L (2012) Immuno-modulatory effects of vitamin D3 in human monocyte and macrophages. Cell Immunol 280:36-43. https://doi.org/10.1016/j.cellimm.2012.10.009

13. Tabatabaeizadeh SA, Avan A, Bahrami A, Khodashenas E, Esmaeili H, Ferns GA, Abdizadeh MF, Ghayour-Mobarhan M (2017) High dose supplementation of vitamin D affects measures of systemic inflammation: reductions in high sensitivity C-reactive protein level and neutrophil to lymphocyte ratio (NLR) distribution. J Cell Biochem 118:4317-4322. https://doi.org/10.1002/jcb. 26084

14. Liefaard MC, Ligthart S, Vitezova A, Hofman A, Uitterlinden AG, Kiefte-de Jong JC, Franco OH, Zillikens MC, Dehghan A (2015) Vitamin D and C-reactive protein: a Mendelian randomization study. PLoS ONE 6(10):e0131740. https://doi.org/10.1371/journ al.pone. 0131740

15. Tao RX, Zhou QF, Xu ZW, Hao JH, Huang K, Mou Z, Jiang XM, Tao FB, Zhu P (2015) Inverse correlation between vitamin D and C-reactive protein in newborns. Nutrients 10(7):9218-9228. https://doi.org/10.3390/nu7115468

16. Park YC, Kim J, Seo MS, Hong SW, Cho ES, Kim JK (2017) Inverse relationship between vitamin D levels and platelet indices in Korean adults. Hematology 22:623-629. https://doi.org/10. 1080/10245332.2017.1318334

17. Hélou J, Moutran R, Maatouk I, Haddad F (2013) Raynaud's phenomenon and vitamin D. Rheumatol Int 33:751-755. https://doi. org/10.1007/s00296-012-2445-x

18. Szymańska-Kałuża J, Biernacka-Zielińska M, Stańczyk J, Smolewska E (2013) Vitamin D level in children with juvenile idiopathic arthritis and its correlation with clinical picture of the disease. Reumatologia 51:271-276. https://doi.org/10.5114/reum. 2013.37250

19. Saggese G, Vierucci F, Boot AM, Czech-Kowalska J, Weber G, Camargo CA Jr, Mallet E, Fanos M, Shaw NJ, Holick MF (2015) Vitamin D in childhood and adolescence: an expert position statement. Eur J Pediatr 174:565-576. https://doi.org/10.1007/ s00431-015-2524-6

20. Shagan BP, Friedman SA (1976) Raynaud's phenomenon in hypothyroidism. Angiology 27:19-25. https://doi.org/10.1177/00033 1977602700104

21. Lateiwish AM, Fehér J, Baraczka K, Rácz K, Kiss R, Gláz E (1992) Remission of Raynaud's phenomenon after L-thyroxine therapy in a patient with hypothyroidism. J Endocrinol Invest 15:49-51. https://doi.org/10.1007/BF03348657

22. Shagan BP, Friedman SA (1980) Raynaud's phenomenon and thyroid deficiency. Arch Intern Med 140:832-833

23. Withrington RH, Seifert MH (1981) Hypothyroidism associated with mixed connective tissue disease and its response to steroid therapy. Ann Rheum Dis 40:315-316. https://doi.org/10.1136/ard. 40.3.315

24. Ciaffi J, Ajasllari N, Mancarella L, Brusi V, Meliconi R, Ursini F (2020) Nailfold capillaroscopy in common non-rheumatic conditions: a systematic review and applications for clinical practice.
Microvasc Res 131:104036. https://doi.org/10.1016/j.mvr.2020. 104036

25. Ingegnoli F, Ughi N, Dinsdale G, Orenti A, Boracchi P, Allanore Y, Foeldvari I, Sulli A, Cutolo M, Smith V, Herrick AL, EULAR Study Group on Microcirculation in RheumaticDiseases (2017) An international SUrvey on non-iNvaSive tecHniques to assess the mIcrocirculation in patients with RayNaud's phEnomenon (SUNSHINE survey). Rheumatol Int 37:1879-1890. https://doi. org/10.1007/s00296-017-3808-0

26. Tavakol ME, Fatemi A, Karbalaie A, Emrani Z, Erlandsson BE (2015) Nailfold capillaroscopy in rheumatic diseases: which parameters should be evaluated? Biomed Res Int 2015:974530. https://doi.org/10.1155/2015/974530

27. Schonenberg-Meinema D, Bergkamp SC, Nassar-Sheikh Rashid A, van der Aa LB, de Bree GJ, Ten Cate R, Cutolo M, Hak AE, Muller PCH, van Onna M, Kuijpers TW, Smith V, van den Berg JM (2021) Nailfold capillary abnormalities in childhood-onset systemic lupus erythematosus: a cross-sectional study compared with healthy controls. Lupus 30(5):818-827. https://doi.org/10. $1177 / 0961203321998750$

28. Torrente-Segarra V, Hernández-Baldizón S, Mosquera JM, Antón J (2021) The importance of nailfold capillaroscopy in children with rheumatic diseases. Clin Exp Rheumatol 39(Suppl 128-1):35

29. Okazaki R, Ozono K, Fukumoto S, Inoue D, Yamauchi M, Minagawa M, Michigami T, Takeuchi Y, Matsumoto T, Sugimoto T (2017) Assessment criteria for vitamin D deficiency/insufficiency in Japan: proposal by an expert panel supported by the Research Program of Intractable Diseases, Ministry of Health, Labour and Welfare, Japan, the Japanese Society for Bone and Mineral Research and the Japan Endocrine Society [Opinion]. J Bone Miner Metab 235:1-5. https://doi.org/10.1007/s00774-016-0805-4

30. Rossi D, Russo A, Manna E, Binello G, Baldovino S, Sciascia S, Roccatello D (2013) The role of nail-videocapillaroscopy in early diagnosis of scleroderma. Autoimmun Rev 12:821-825. https:// doi.org/10.1016/j.autrev.2012.11.006

31. Tamez-Rivera O, Villarreal-Treviño AV, Castañeda-Macazaga T, Britton-Robles SC, Ramos-Gómez LI, Rubio-Pérez NE (2021) Abnormal nailfold capillaroscopy in a patient with multisystem inflammatory syndrome in children. Pediatr Infect Dis J 40(3):e113-e115. https://doi.org/10.1097/INF.0000000000 003022

32. Sampaio-Barros MM, Takayama L, Sampaio-Barros PD, Bonfá E, Pereira RM (2016) Low vitamin D serum levels in diffuse systemic sclerosis: a correlation with worst quality of life and severe capillaroscopic findings. Rev Bras Reumatol Engl Ed 56:337-344. https://doi.org/10.1016/j.rbre.2016.05.006 (English, Portuguese)

33. Trombetta AC, Smith V, Gotelli E, Ghio M, Paolino S, Pizzorni C, Vanhaecke A, Ruaro B, Sulli A, Cutolo M (2017) Vitamin D deficiency and clinical correlations in systemic sclerosis patients: a retrospective analysis for possible future developments. PLoS ONE 9(12):e0179062. https://doi.org/10.1371/journal.pone.01790 62

34. Groseanu L, Bojinca V, Gudu T, Saulescu I, Predeteanu D, Balanescu A, Berghea F, Opris D, Borangiu A, Constantinescu C, Negru M, Ionescu R (2016) Low vitamin D status in systemic sclerosis and the impact on disease phenotype. Eur J Rheumatol 3:50-55. https://doi.org/10.5152/eurjrheum.2015.0065

35. Gupta S, Goyal P, Feinn RS, Mattana J (2019) Role of vitamin D and its analogues in diabetic nephropathy: a meta-analysis. Am J Med Sci 357:223-229. https://doi.org/10.1016/j.amjms.2018.12. 005

36. Shillo P, Selvarajah D, Greig M, Gandhi R, Rao G, Wilkinson ID, Anand P, Tesfaye S (2019) Reduced vitamin D levels in painful diabetic peripheral neuropathy. Diabet Med 36:44-51. https://doi. org/10.1111/dme.13798 
37. Tecilazich F, Formenti AM, Giustina A (2020) Role of vitamin D in diabetic retinopathy: pathophysiological and clinical aspects. Rev Endocr Metab Disord 7:1-13. https://doi.org/10.1007/ s11154-020-09575-4

38. Harris RA, Pedersen-White J, Guo DH, Stallmann-Jorgensen IS, Keeton D, Huang Y, Shah Y, Zhu H, Dong Y (2011) Vitamin D3 supplementation for 16 weeks improves flow-mediated dilation in overweight African-American adults. Am J Hypertens 24:557562. https://doi.org/10.1038/ajh.2011.12

39. Molnár I, Czirják L (2000) Euthyroid sick syndrome and inhibitory effect of sera on the activity of thyroid 5'-deiodinase in systemic sclerosis. Clin Exp Rheumatol 18:719-724

40. Gordon MB, Klein I, Dekker A, Rodnan GP, Medsger TA Jr (1981) Thyroid disease in progressive systemic sclerosis: increased frequency of glandular fibrosis and hypothyroidism. Ann Intern Med 95:431-435. https://doi.org/10.7326/ 0003-4819-95-4-431

41. De Martinis M, Sirufo MM, Ginaldi L (2018) Raynaud's phenomenon and nailfold capillaroscopic findings in anorexia nervosa. Curr Med Res Opin 34:547-550. https://doi.org/10.1080/03007 995.2017.1417828

Publisher's Note Springer Nature remains neutral with regard to jurisdictional claims in published maps and institutional affiliations. 